\title{
Periodontal health related-inflammatory and metabolic profiles of patients with end-stage renal disease: potential strategy for predictive, preventive, and personalized medicine
}

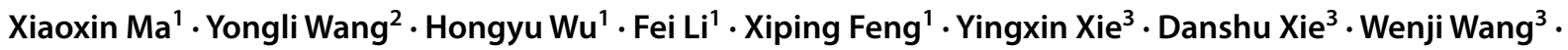 \\ Edward Chin Man Lo ${ }^{4}(1) \cdot$ Haixia Lu' ${ }^{1}$
}

Received: 4 February 2021 / Accepted: 10 March 2021 / Published online: 22 April 2021

(c) The Author(s) 2021

\begin{abstract}
Objectives To compare the periodontal health related-inflammatory and metabolic differences between patients with endstage renal disease (ESRD) and healthy controls (HC), and to identify potential biomarkers in gingival crevicular fluid (GCF) and serum of ESRD patients for predictive, preventive, and personalized medicine (PPPM).

Methods Patients with ESRD (ESRD group; $n=52$ ) and healthy controls (HC group; $n=44$ ) were recruited. Clinical periodontal parameters were recorded. The differential metabolites in the GCF and serum were identified by liquid chromatography/mass spectrometry (LC/MS). Inflammatory markers including interleukin-1 $\beta$ (IL-1 $\beta$ ), interleukin-6 (IL-6), interleukin-8 (IL-8), and C-reactive protein (CRP) were also assessed.

Results In the ESRD group, IL-8 and CRP were significantly higher in GCF, whereas IL-6 and CRP were significantly higher in serum, compared with $\mathrm{HC}$ group (all $P<0.05$ ). In the case of GCF, taurine levels were positively correlated with IL-8 levels in both groups (all $P<0.05$ ). In the case of serum, L-phenylalanine and $p$-hydroxyphenylacetic acid levels were positively correlated with CRP levels in both groups (all $P<0.05$ ). Significant positive correlations were observed between metabolites (including pseudouridine, L-phenylalanine, and $p$-hydroxyphenylacetic acid) and IL-6 levels only in ESRD group. Conclusions IL-8 and CRP are potential inflammatory makers that reflect the periodontal health of ESRD individual, which may be considered the valuable predictive diagnostics in the agreement with PPPM philosophy. Besides, metabolites of taurine in GCF as well as L-phenylalanine and $p$-hydroxyphenylacetic acid in serum are possible biomarkers correlated with inflammatory markers. All these biomarkers may also be highly recommended as a novel predictive/diagnostic tool for the assessment of inflammatory status from the perspectives of PPPM in view of susceptible population and individual screening.
\end{abstract}

Keywords Predictive preventive personalized medicine $\cdot$ Chronic kidney disease $\cdot$ End-stage renal disease $\cdot$ Diseasespecific metabolic profile $\cdot$ Mass spectrometry $\cdot$ Gingival crevicular fluid $\cdot$ Interleukin- $8 \cdot$ Interleukin- $6 \cdot$ C-reactive protein $\cdot$ Serum $\cdot$ Periodontal health $\cdot$ Metabolites $\cdot$ Inflammation $\cdot$ COVID-19

Xiaoxin Ma and Yongli Wang contributed equally to this work.

Xiaoxin Ma and Yongli Wang share the first authorship.

Haixia Lu

ritalu0225@hotmail.com

1 Department of Preventive Dentistry, Shanghai Ninth People's Hospital, College of Stomatology, Shanghai Jiao Tong University School of Medicine, National Clinical Research Center for Oral Diseases, Shanghai Key Laboratory of Stomatology, 639 Zhizaoju Road, Shanghai, China

2 Laboratory of Oral Microbiota and Systemic Diseases, Shanghai Ninth People's Hospital, College of Stomatology,
Shanghai Jiao Tong University School of Medicine, National Clinical Research Center for Oral Diseases, Shanghai Key Laboratory of Stomatology, Shanghai, China

3 Department of Nephrology, Shanghai Ninth People's Hospital, College of Stomatology, Shanghai Jiao Tong University School of Medicine, 639 Zhizaoju Road, Shanghai, China

4 Dental Public Health, Faculty of Dentistry, University of Hong Kong, 34 Hospital Road, Hong Kong, China 


$\begin{array}{ll}\text { Abbreviations } \\ \text { PPPM } & \begin{array}{l}\text { Predictive, preventive, and personalized } \\ \text { medicine }\end{array} \\ \text { GCF } & \begin{array}{l}\text { Gingival crevicular fluid } \\ \text { ESRD }\end{array} \\ \text { End-stage renal disease } \\ \text { HCs } & \text { Healthy controls } \\ \text { IL } & \text { Interleukin } \\ \text { CRP } & \text { C-Reactive protein } \\ \text { PDD } & \text { Periodontal pocket depth } \\ \text { GBI } & \text { Gingival bleeding index } \\ \text { CI } & \text { Calculus index } \\ \text { LC/MS } & \text { Liquid chromatography/mass spectrometry } \\ \text { IQR } & \text { Interquartile range } \\ \text { PCA } & \text { Principal component analysis } \\ \text { OPLS-DA } & \text { Orthogonal partial least-squares discriminant } \\ & \text { analysis } \\ \text { FC } & \text { Fold change } \\ \text { AUC } & \text { Area under the curve } \\ \text { COVID-19 } & \text { Coronavirus disease 2019 }\end{array}$

\section{Introduction}

\section{End-stage renal disease as the healthcare challenge}

End-stage renal disease (ESRD) refers to the end stage of chronic kidney disease (CKD) that requires renal replacement therapy, including peritoneal dialysis, hemodialysis, or kidney transplantation [1]. ESRD patients with hemodialysis therapy have a relatively lower life expectancy than the general population, whose 5-year survival rate is estimated to be $76 \%$ [2]. Evidence from observational studies suggests that a larger proportion of patients undergoing dialysis treatment suffer from periodontal diseases [3]. And it is reported that $58.9 \%$ of hemodialysis patients have moderate to severe periodontitis [4]. Currently, a number of studies have shown the association between periodontal health and ESRD [4-7].

\section{End-stage renal disease as a risk factor of periodontal health}

According to a recent systematic review, there are conflicting conclusions about the reciprocal relationship between CKD and periodontal disease, and the evidence that CKD has an impact on periodontal status is weak [7]. Immune response to the etiological factors of periodontal diseases is the determinant of disease susceptibility. ESRD patients are more susceptible to periodontal disease due to general debilitation and depression of the immunological system. The altered saliva composition and persistent low-grade inflammation in patients with CKD also mechanistically influence the onset and/or progression of periodontal disease $[8,9]$. In the progression of CKD, more cytokines were secreted by inflammatory cells to the inflammation sites, which aggravates the periodontal disease [10, 11]. CKD, especially ESRD, may affect periodontal condition through systemic immune disorders, decreased saliva secretion, and accumulation of waste products in the blood [12-15].

\section{IL-8 as an indicator of periodontal health status}

Studies based on GCF analysis exhibit the examination of the levels of inflammatory chemokines such as IL- 8 can be used to assess periodontal health status [16-18]. IL-8 is an important chemokine and can be produced by many cell types in gingival epithelial tissues such as endothelial cells and neutrophils $[19,20]$. Higher expression of IL- 8 can be detected when the gingival tissue is in an inflammatory state in comparison to healthy gingiva [21,22].

\section{The role of metabolites in pathogenesis of periodontal disease}

In addition to inflammatory chemokines as the biological markers to the periodontal inflammation, the dynamics of the metabolic composition in GCF also hints the development of periodontal disease. Previous studies have reported that significant differences in metabolites related to inflammation, oxidative stress, tissue degradation, and bacterial metabolism could be observed in GCF between the population with and without periodontitis [23, 24]. Oxidative stress is a potential mediator of the progression of periodontal disease [25]. Some metabolic pathways such as purine metabolism serve as an indication of an increased inflammatory response and are associated with oxidative stress [26]. Glucose metabolism is reported to play an important role in the oral bacterial metabolism [27]. Metabolites can not only reflect metabolic activities but also affect clinical phenotypes, as they are the end products of biological processes and indicate the expression levels of many functional genes and proteins [28]. Metabolite changes in GCF could serve as a useful tool to reflect the periodontal inflammation status.

\section{Working hypothesis}

Considering these previous observations, the levels of certain metabolites may be associated with inflammatory levels in both GCF and serum. Inflammatory markers can be an effective diagnostic tool to assess the periodontal status of individuals, especially of the ESRD population. And the change of metabolites can serve as novel predictive/diagnostic tool for periodontal health. Therefore, the aim of present study was to compare the periodontal health-related inflammatory markers and metabolite levels in serum and GCF between ESRD group and $\mathrm{HC}$ group using metabolomics, and to explore the association between inflammatory markers and metabolites. 
Integration of inflammatory and metabolomic data with clinical data will provide more valuable information for PPPM in GCF and serum of the individuals, especially of ESRD patients.

\section{Materials and methods}

\section{Participants}

ESRD patients undergoing hemodialysis were enrolled from Department of Nephrology, Shanghai Ninth People's Hospital, Shanghai, China, and healthy individuals (healthy controls, HC group) were recruited from the Physical Examination Center of the same hospital from February 2019 to June 2020. The inclusion criteria for the ESRD group were age between 20 and 75 years and diagnosis of ESRD with a stable hemodialysis status. The exclusion criteria were as follows: having taken periodontal treatment within the last 6 months, antibiotic intake within 3 months, pregnancy or lactating status, presence of less than 15 natural teeth excluding third molars, presence of other systemic diseases except hypertension and diabetes (e.g., rheumatoid arthritis, systemic lupus erythematosus, psoriasis) or other severe systemic infections, and immunosuppressive therapy within last 30 days. The $\mathrm{HC}$ group were participants who were systemically healthy without kidney diseases. The other inclusion and exclusion criteria were the same as those for the ESRD group. All healthy controls were age-matched and sex-matched with patients undergoing hemodialysis.

Prior to the implementation of the study, a full-mouth periodontal examination was performed on all participants by an examiner using a sterile periodontal probe (PCPUNC 15, Hu-Friedy, Chicago, IL, USA). Periodontal pocket depth (PPD), gingival bleeding index (GBI), and calculus index (CI) were recorded to identify the periodontal health status. Structured questionnaire was used to collect the information on sociodemographic background (age and sex), smoking habit, oral health-related behaviors (toothbrushing frequency and use of dental floss), and medical history (presence of hypertension or diabetes) of each participant.

Ethical approval was obtained from the Research Ethics Committee of Shanghai Ninth People's Hospital. Each participant was informed about the purpose and procedure of the study. Each participant signed a written informed consent prior to the implementation of the study.

\section{Collection of biological samples}

\section{Serum}

A total of $3 \mathrm{ml}$ peripheral blood was collected in vacutainer tubes in the morning from all participants. The blood samples were incubated at room temperature for not more than $2 \mathrm{~h}$ and centrifuged for $10 \mathrm{~min}$ at $2000 \times g$ at $4{ }^{\circ} \mathrm{C}$. Serum was collected in the empty tubes and then stored at $-80^{\circ} \mathrm{C}$.

\section{GCF}

All participants were instructed not to eat or drink within $2 \mathrm{~h}$ prior to the collection of GCF. PerioPaper Strips ${ }^{\circledR}$ (Oraflow Inc., New York, USA) were used to collect GCF samples from the disto-buccal and mesio-buccal sites of all the first molars. The targeted area was separated with cotton rolls, PerioPaper Strips ${ }^{\circledR}$ were placed in the gingival sulcus for $30 \mathrm{~s}$ to collect GCF, and all periopaper strips from different sites were pooled in the same tube. The samples were then stored in an empty tube at $-80^{\circ} \mathrm{C}$.

\section{Estimation of inflammatory markers in GCF and serum}

According to the kit instructions, the concentrations of IL-1 $\beta$, IL-6, and IL-8 in GCF and serum as well as CRP in GCF were measured using Human High-Sensitivity T Cell Magnetic Bead Panel (Luminex Corporation, Austin, TX, USA). Furthermore, the concentration of CRP in serum was measured using a CRP ELISA kit (R\&D Systems, Minneapolis, MN, USA).

\section{Metabolite extraction}

For metabolite extraction from GCF, $200 \mu$ l extraction solution (methanol:acetonitrile: water $=2: 2: 1$, with isotopically labeled internal standard mixture) was added to periopaper strips. The mixture was vortexed for $30 \mathrm{~s}$ and ground at $35 \mathrm{~Hz}$ for $4 \mathrm{~min}$. Thereafter, the samples were sonicated for $15 \mathrm{~min}$ in ice-water bath. For the extraction of serum metabolites, $50 \mu \mathrm{l}$ of sample was mixed with $200 \mu \mathrm{l}$ of extraction solution (acetonitrile:methanol $=1: 1$, containing isotopically labeled internal standard mixture). The mixture was vortexed for $30 \mathrm{~s}$ and sonicated for $10 \mathrm{~min}$ in an ice-water bath. Both mixtures were incubated for $1 \mathrm{~h}$ at $-40^{\circ} \mathrm{C}$ and centrifuged at $12,000 \mathrm{rpm}$ for $15 \mathrm{~min}$ at $4{ }^{\circ} \mathrm{C}$. The supernatant was separated and mixed with quality control (QC) samples for machine testing.

\section{Metabolomics analysis}

LC/MS was used for metabolomics analysis. It was performed using a UHPLC system (Vanquish, Thermo Fisher Scientific) containing a UPLC BEH Amide column $(2.1 \mathrm{~mm} \times 100 \mathrm{~mm}, 1.7 \mu \mathrm{m})$ with Q Exactive HFX mass spectrometer (Orbitrap MS, Thermo). HPLC contained aqueous phase $(25 \mathrm{mmol} / \mathrm{l}$ ammonium acetate and $25 \mathrm{mmol} / \mathrm{l}$ ammonia) and acetonitrile. The analysis was processed with elution gradient. Other LC parameters were as follows: 
mobile phase flow rate: $0.5 \mathrm{ml} / \mathrm{min}$, column temperature: $30{ }^{\circ} \mathrm{C}$, sample pan temperature: $4{ }^{\circ} \mathrm{C}$, and injection volume: $4 \mu \mathrm{l}$. The QE HFX mass spectrometer was used to collect primary and secondary mass spectrometry data under the control of the acquisition software (Xcalibur, Thermo).

\section{Data preprocessing and annotation}

The raw data was transformed into the mzXML format, using the R software to process peak detection, extraction, alignment, and integration. Furthermore, an in-house MS2 database (BiotreeDB) was used for metabolite annotation with the cutoff for annotation set at 0.3 .

\section{Sample size}

A pilot study ( $n=15$ in each group) was performed to obtain information about inflammatory markers. The levels of IL-8 were significantly different between two groups, which we chose as the primary outcome. We assumed twosided hypothesis testing under the 5\% type I error and $90 \%$ statistical power to detect a difference of $0.815 \mathrm{ng} / \mathrm{ml}$ with estimated group standard deviations of $1.331 \mathrm{ng} / \mathrm{ml}$ and $0.603 \mathrm{ng} / \mathrm{ml}$. A total of 36 participants were required in each group, which was calculated using PASS 11.0 (NCSS, LLC. Kaysville, Utah, USA).

\section{Statistical data analysis}

The Kolmogorov-Smirnov test was performed to test the normality of data distribution. Numerical variables were presented as mean (SD) when parameters were normally distributed or as median (interquartile range, IQR) when the distribution was skewed. Student's $t$-test or Mann-Whitney $U$ test was used to compare the differences between ESRD and HC groups depending on whether variances were homogenous and normal. Chi-square test or Fisher's exact test was used to compare the differences of sociodemographic background, lifestyle, and medical history between the two groups. Based on the SIMCA software V16.0.2 (Sartorius Stedim Data Analytics AB, Umea, Sweden), principal component analysis (PCA) was used to show the sample distribution within the two groups. Orthogonal partial least-squares discriminant analysis (OPLS-DA) was also used to assess total differences of metabolites between the two groups. Multivariate models were performed to determine various metabolites with variable importance (VIP) values $>1.0$ and $P<0.05$ as the key metabolites for a further analysis. Pearson correlation coefficient was used to calculate the correlations between metabolites and inflammatory markers. Multiple factor ANOVA was used to assess the association between system diseases and the levels of IL-8 in the CGF. $P<0.05$ was considered to be statistically significant.

\section{Results}

\section{Participant characteristics}

A total of 96 participants were enrolled in the study. Among these, 52 were ESRD patients receiving hemodialysis treatment ( 30 males and 22 females; group designated as ESRD), and 44 were healthy controls (25 males and 19 females; group designated as HC). Characteristics of all patients are summarized in Table 1 . There were no significant differences regarding age, smoking history, and oral health-related behaviors between the two groups (all $P>0.05$ ). The proportions of participants with diabetes and hypertension were significantly higher in the ESRD group than in the $\mathrm{HC}$ group (all $P<0.05$ ). There were significant differences in periodontal parameters including PPD, GBI, and CI between two groups (all $P<0.05$ ).

\section{Comparison of inflammatory markers in serum and GCF}

The levels of IL-8 and CRP of GCF were significantly higher in the ESRD group than in the HC group $(P=0.032$ and $P<0.001$, respectively). In addition, the levels of IL-6 and CRP of serum were higher in the ESRD group $(P=0.008$ and $P<0.001$, respectively). However, there were no significant differences on the IL- $1 \beta$ and IL- 6 levels of GCF between two groups. Moreover, IL-1 $\beta$ and IL-8 levels in serum did not show significant differences between the two groups (Table 2).

\section{Results of multiple factor ANOVA of the level of IL-8 in the GCF}

Since the distributions of diabetes and hypertension history between ESRD and HC groups were significantly different, multiple factor AVOVA was performed to explore the association between groups (ESRD and HC groups), diabetes, hypertension history, and IL-8 levels in the GCF. As shown in Table 3, only the group was significantly associated with the IL-8 levels of GCF ( $\beta=1.17 ; P=0.001)$, while diabetes history and hypertension history were not associated with IL-8 level $(\beta=0.27, P=0.486$ and $\beta=-0.61, P=0.071)$.

\section{Metabolomics analysis of serum samples}

A total of 261 differential metabolites with VIP $>1$ and $P<0.05$ were identified in the serum samples for the hierarchical cluster analysis (Fig. 1a). In the plot of PCA scores, the obvious divergence of metabolites in serum samples could be observed between ESRD group and HC group (Fig. 1b). The OPLS-DA model (Fig. 1c) was constructed 
Table 1 Participant characteristics of the ESRD and $\mathrm{HC}$ groups

\begin{tabular}{|c|c|c|c|c|}
\hline & & ESRD group & HC group & $P$ value \\
\hline Total $; \mathrm{n}$ & & 52 & 44 & \\
\hline \multirow[t]{5}{*}{ Age groups; $n(\%)$} & & & & 0.574 \\
\hline & $\leq 40$ & $4(7.7 \%)$ & $5(11.4 \%)$ & \\
\hline & $41-50$ & $10(19.2 \%)$ & $11(25.0 \%)$ & \\
\hline & $51-60$ & $17(32.7 \%)$ & $16(36.4 \%)$ & \\
\hline & $\geq 61$ & $21(40.4 \%)$ & $12(27.2 \%)$ & \\
\hline \multirow[t]{3}{*}{ Sex; $n(\%)$} & & & & 0.548 \\
\hline & Male & $30(57.7 \%)$ & $25(56.8 \%)$ & \\
\hline & Female & $22(42.3 \%)$ & $19(43.2 \%)$ & \\
\hline \multirow[t]{3}{*}{ Smoking history; $n(\%)$} & & & & 0.462 \\
\hline & Yes & $15(28.8 \%)$ & $14(31.8 \%)$ & \\
\hline & No & $37(71.2 \%)$ & $30(68.2 \%)$ & \\
\hline \multirow[t]{3}{*}{ Toothbrushing frequency } & & & & 0.064 \\
\hline & $\leq 1$ per day & $25(48.1 \%)$ & $13(29.5 \%)$ & \\
\hline & $\geq 2$ per day & $27(51.9 \%)$ & $31(70.5 \%)$ & \\
\hline \multirow[t]{3}{*}{ Use of dental floss } & & & & 0.222 \\
\hline & Yes & $5(9.6 \%)$ & $8(18.2 \%)$ & \\
\hline & No & $47(90.4 \%)$ & $36(81.8 \%)$ & \\
\hline \multirow[t]{3}{*}{ Diabetes history*; $n(\%)$} & & & & 0.019 \\
\hline & Yes & $13(25.0 \%)$ & $3(7.0 \%)$ & \\
\hline & No & $39(75.0 \%)$ & $40(93.0 \%)$ & \\
\hline \multirow[t]{3}{*}{ Hypertension history*; $n(\%)$} & & & & $<0.001$ \\
\hline & Yes & $40(76.9 \%)$ & $10(23.3 \%)$ & \\
\hline & No & $12(23.1 \%)$ & $33(76.7 \%)$ & \\
\hline GBI (\%), median (IQR) & & $20.60(17.86-23.68)$ & $14.29(12.96-17.86)$ & 0.008 \\
\hline CI (\%), median (IQR) & & $10.79(7.41-12.00)$ & $7.28(3.70-10.71)$ & 0.013 \\
\hline PPD (mm), median (IQR) & & $3.46(3.33-3.62)$ & $3.32(3.19-3.44)$ & 0.020 \\
\hline
\end{tabular}

$E S R D$, end-stage renal disease; $H C$, healthy controls; $P P D$, periodontal pocket depth; $G B I$, gingival bleeding index; $C I$, calculus index; $I Q R$, interquartile range

* One missing value

many times to obtain the $R^{2} Y$ and $Q^{2}$ values of the random model $\left(R^{2} Y=0.983\right.$ and $\left.Q^{2}=0.973\right)$, demonstrating that the original model had a good robustness and there was no overfitting (Fig. 1d). As shown in Table 4, the four main metabolic pathways that differed dramatically in serum included arginine and proline metabolism, phenylalanine metabolism, glycine/serine/threonine metabolism, and pyrimidine metabolism. Among these various pathways, 19 key metabolites were selected for further analysis, and fold changes (FCs) of key metabolites $(P<0.05)$ were calculated.
Table 2 Comparison of inflammatory markers between the ESRD and $\mathrm{HC}$ groups

\begin{tabular}{lccr}
\hline Inflammatory markers & $\begin{array}{l}\text { ESRD group } \\
\text { Median (IQR) }\end{array}$ & $\begin{array}{l}\text { HC group } \\
\text { Median (IQR) }\end{array}$ & $P$ value \\
\hline Serum & & & \\
$\quad$ IL-1 $\beta(\mathrm{pg} / \mathrm{ml})$ & $0.75(0.60-1.01)$ & $0.88(0.60-1.25)$ & 0.225 \\
IL-6 (pg/ml) & $5.76(4.89-7.68)$ & $4.46(3.36-5.03)$ & 0.008 \\
IL-8 (pg/ml) & $46.55(38.98-82.49)$ & $38.86(32.29-47.41)$ & 0.073 \\
CRP $(\mathrm{ng} / \mathrm{ml})$ & $2.20(1.60-4.09)$ & $0.74(0.50-1.26)$ & $<0.001$ \\
GCF & & & 0.186 \\
IL-1 $\beta(\mathrm{ng} / \mathrm{ml})$ & $0.26(0.20-0.37)$ & $0.34(0.20-0.53)$ & 0.622 \\
IL-6 $(\mathrm{pg} / \mathrm{ml})$ & $2.75(2.14-3.23)$ & $2.46(2.19-3.24)$ & 0.032 \\
IL-8 $(\mathrm{ng} / \mathrm{ml})$ & $1.44(0.81-2.26)$ & $0.88(0.55-1.23)$ & $<0.001$ \\
CRP $(\mathrm{ng} / \mathrm{ml})$ & $0.48(0.29-0.78)$ & $0.16(0.11-0.23)$ & \\
\hline
\end{tabular}

$E S R D$, end-stage renal disease; $H C$, healthy controls; $I L-1 \beta$, interleukin- $1 \beta ; I L-6$, interleukin-6; $I L-8$, interleukin-8; $C R P, \mathrm{C}$-reactive protein; $I Q R$, interquartile range 
Table 3 Results of multiple factor ANOVA analysis of the level of IL-8 in the GCF

\begin{tabular}{lllll}
\hline Variables & & Beta & $95 \%$ CI & $P$ value \\
\hline Group & ESRD & 1.17 & $0.49-1.84$ & 0.001 \\
& $\mathrm{HC}^{\mathrm{a}}$ & & & \\
Diabetes & Yes & 0.27 & $-0.5-1.04$ & \\
& $\mathrm{No}^{\mathrm{a}}$ & & & \\
\multirow{2}{*}{ Hypertension } & & & & 0.486 \\
& Yes & -0.61 & $-1.28-0.05$ & \\
& $\mathrm{No}^{\mathrm{a}}$ & & &
\end{tabular}

$R^{2}=0.130$

$G C F$, gingival crevicular fluid; $I L-8$, interleukin-8; $E S R D$, end-stage renal disease; $H C$, healthy controls; $C I$, confidence interval

${ }^{\mathrm{a}}$ Reference group

The metabolites including 4-hydroxybenzoic acid, 4-acetamidobutanoic acid, and phenylacetylglutamine $\left(\log _{2}\right.$ (FC): $7.287,7.456$, and 7.459, respectively) showed the highest FCs in the ESRD group in comparison with HC group. To assess the discriminating accuracy of the metabolites in differentiating two populations, the area under curve (AUC) was employed to identify 19 metabolites of serum. And the high AUCs of metabolites were observed, most of which were up to 1.00 .

\section{Metabolomics analysis of GCF samples}

A total of 88 differential metabolites with VIP $>1$ and $P<0.05$ were identified for the hierarchical cluster analysis (Fig. 2a). The PCA score showed a good separation of metabolites in GCF between the two groups (Fig. 2b). The OPLS-DA model (Fig. 2c) was constructed many times to obtain the $R^{2} Y$ and $Q^{2}$ values of the random model $\left(R^{2} Y=0.682\right.$ and $Q^{2}=0.616$, Fig. 2d). As shown in Table 5, the seven main metabolic pathways that showed the significant differences in GCF samples were galactose metabolism, nicotinate and nicotinamide metabolism, phenylalanine metabolism, purine metabolism, starch and sucrose metabolism, taurine and hypotaurine metabolism, and pyrimidine metabolism based on the KEGG pathway enrichment analysis $(P<0.05)$. The metabolites significantly upregulated in ESRD group in comparison to HC group were raffinose, a

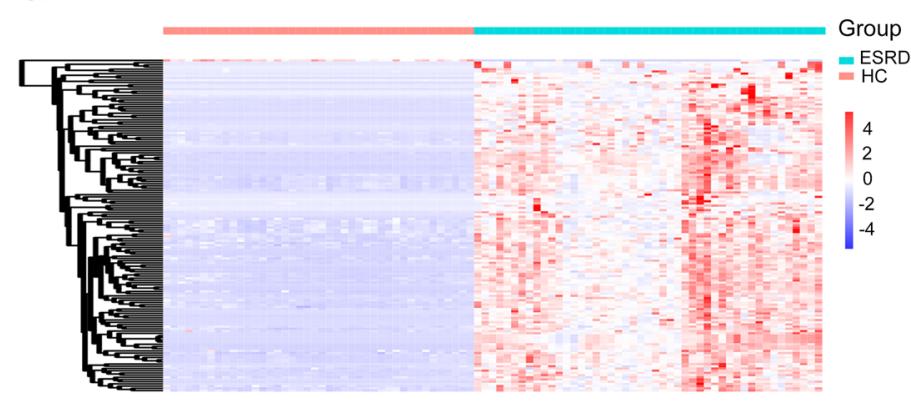

C

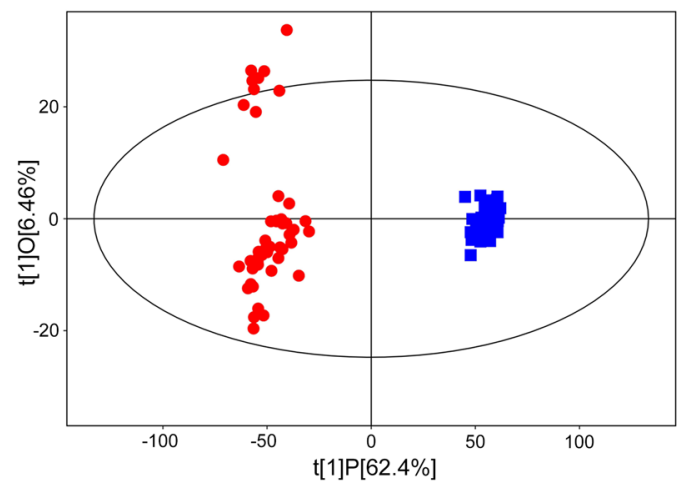

Fig. 1 Metabolic profile of serum based on LC-MS. a Heatmap of metabolites between the ESRD group and the HC group. b PCA score plot model of serum samples. c OPLS-DA score plot of serum samples. d OPLS-DA permutation plot of serum samples. LC-MS,
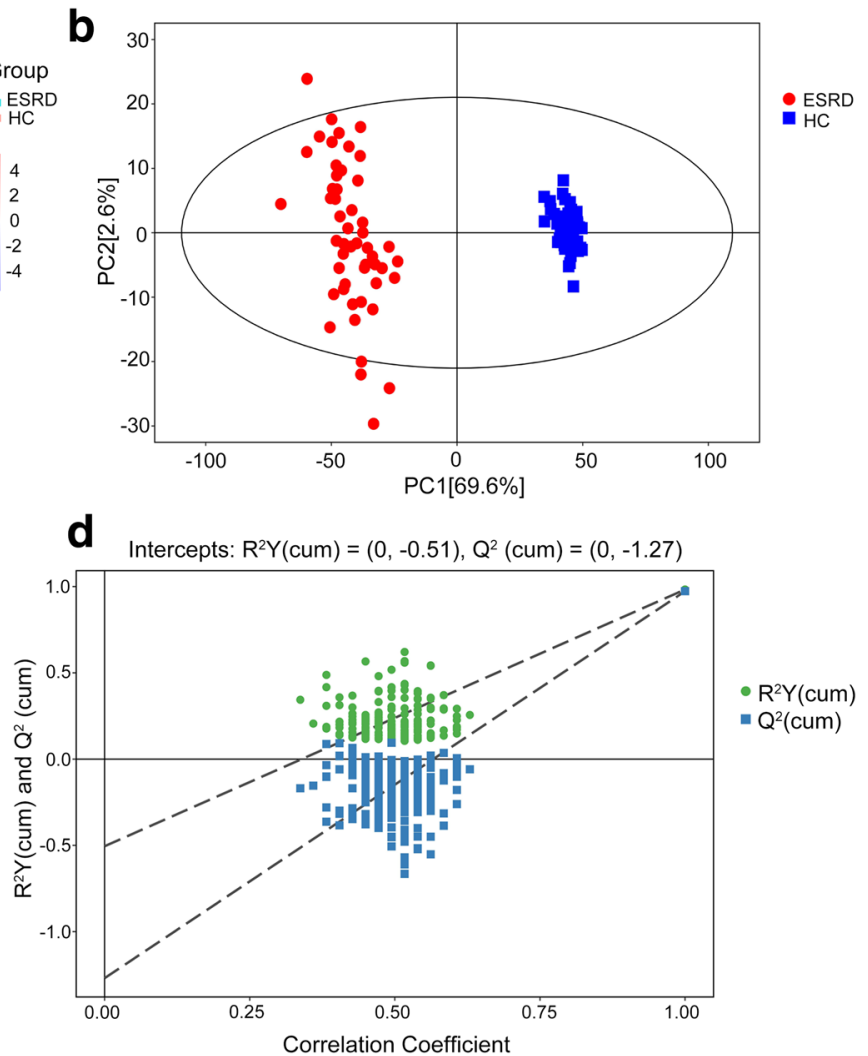

liquid chromatography/mass spectrometry; ESRD, end-stage renal disease; HC, healthy controls; OPLS-DA, orthogonal partial leastsquares discriminant analysis; PCA, principal component analysis 
Table 4 Differential metabolites of serum between patients with ESRD and healthy controls

\begin{tabular}{|c|c|c|c|c|c|c|}
\hline Metabolic Pathway & Metabolites & Mean (ESRD) & $\begin{array}{l}\text { Mean } \\
(\mathrm{HC})\end{array}$ & Fold change & Log_fold change & AUC value \\
\hline \multirow{3}{*}{$\begin{array}{l}\text { Glycine, serine, and threonine } \\
\text { metabolism }\end{array}$} & Glycine & 1.9834 & 0.6171 & 3.214 & 1.684 & 0.972 \\
\hline & L-Serine & 1.3163 & 0.1549 & 8.497 & 3.087 & 0.995 \\
\hline & L-Allothreonine & 3.0007 & 0.4912 & 6.109 & 2.611 & 0.971 \\
\hline \multirow[t]{6}{*}{ Phenylalanine metabolism } & L-Phenylalanine & 46.5813 & 19.7719 & 2.356 & 1.236 & 0.975 \\
\hline & ortho-Hydroxyphenylacetic acid & 15.8486 & 0.3832 & 41.359 & 5.370 & 1.000 \\
\hline & Phenylacetic acid & 15.8486 & 0.3832 & 41.359 & 5.370 & 0.997 \\
\hline & Phenylacetylglutamine & 174.1466 & 0.9897 & 175.951 & 7.459 & 1.000 \\
\hline & 4-Hydroxybenzoic acid & 13.9005 & 0.0890 & 156.181 & 7.287 & 1.000 \\
\hline & $p$-Hydroxyphenylacetic acid & 2.1442 & 0.0570 & 37.632 & 5.234 & 0.993 \\
\hline \multirow[t]{4}{*}{ Pyrimidine metabolism } & Cytosine & 2.0971 & 0.1987 & 10.556 & 3.400 & 1.000 \\
\hline & Deoxyribose 1-phosphate & 542.9885 & 12.8789 & 42.161 & 5.398 & 0.983 \\
\hline & Uracil & 6.0958 & 1.5430 & 3.951 & 1.982 & 0.995 \\
\hline & Pseudouridine & 34.0287 & 1.6156 & 21.063 & 4.397 & 1.000 \\
\hline \multirow[t]{6}{*}{ Arginine and proline metabolism } & Creatinine & 75.2642 & 2.7376 & 27.493 & 4.781 & 1.000 \\
\hline & Citrulline & 4.3835 & 0.0979 & 44.772 & 5.485 & 1.000 \\
\hline & L-Proline & 38.7071 & 12.2175 & 3.168 & 1.664 & 0.975 \\
\hline & 4-Hydroxyproline & 4.1395 & 0.5685 & 7.281 & 2.864 & 0.970 \\
\hline & 4-Acetamidobutanoic acid & 10.1351 & 0.0577 & 175.635 & 7.456 & 1.000 \\
\hline & $N$-Acetylglutamic acid & 0.9101 & 0.0517 & 17.611 & 4.138 & 1.000 \\
\hline
\end{tabular}

$E S R D$, end-stage renal disease; $H C$, healthy controls; $A U C$, area under curve

alpha-D-glucose, D-maltose, dUMP, and N1-methyl-2-pyridone-5-carboxamide. Other compounds, including taurine, 3 -sulfinoalanine, xanthine, inosine, hypoxanthine, $m$-coumaric acid, hydrocinnamic acid, uridine, and niacinamide, were downregulated in the ESRD group. Metabolites with the greatest FCs $(P<0.05)$ that were increased in ESRD group relative to HC group were dUMP, alpha-D-glucose, and raffinose ( $\log _{2}$ (FC): 3.120, 3.164, and 5.017, respectively). Two metabolites-niacinamide and hydrocinnamic acid-with the greatest FCs were decreased $(P<0.05)$ in the ESRD group compared to the $\mathrm{HC}$ group $\left(\log _{2}(\mathrm{FC}):-1.479\right.$ and -1.708 , respectively). The AUCs of 14 metabolites in the GCF can be observed in Table 5, and almost all metabolites were above 0.700 .

\section{Correlation between inflammatory markers and key metabolites in serum and GCF}

The correlations between key metabolites found in serum and GCF and four inflammatory markers including IL- $1 \beta$, IL-6, IL-8, and CRP were calculated. Only statistically significant positive or negative correlations $(P<0.05)$ between the two groups are shown in Table 6 . In the serum samples, the levels of L-phenylalanine and $p$-hydroxyphenylacetic acid were positively correlated with the levels of CRP in both groups but were positively correlated with the levels of IL-6 only in the ESRD group. Significant positive correlation was observed between pseudouridine and IL- 6 only in ESRD group. In the case of GCF samples, the levels of taurine were positively correlated with IL-8 levels in the ESRD group and HC group, whereas 3-sulfinoalanine levels were positively correlated with IL-8 levels only in the ESRD group.

\section{Discussion}

\section{Achievements in the present study}

The significant associations between periodontal health status and ESRD have been investigated among a number of studies [4-7, 29]. It is evident that patients with kidney diseases have poor periodontal health in comparison to healthy individuals $[30,31]$. However, no study could be traced about comparing GCF metabolites between ESRD patients and the healthy as well as the association between metabolites and periodontal inflammation status; thus, the present study was conducted to compare inflammation markers, the significantly altered metabolites, and their potential associations between two groups. In the present study, we found that IL-8 and CRP levels of GCF in ESRD group were significantly higher than those in the HC group, in which IL- 8 was positively correlated with taurine and 3 -sulfinoalanine. Meanwhile, we observed the periodontal parameters (PPD, GBI, and CI) of ESRD patients were worse than that of healthy participants. 
a

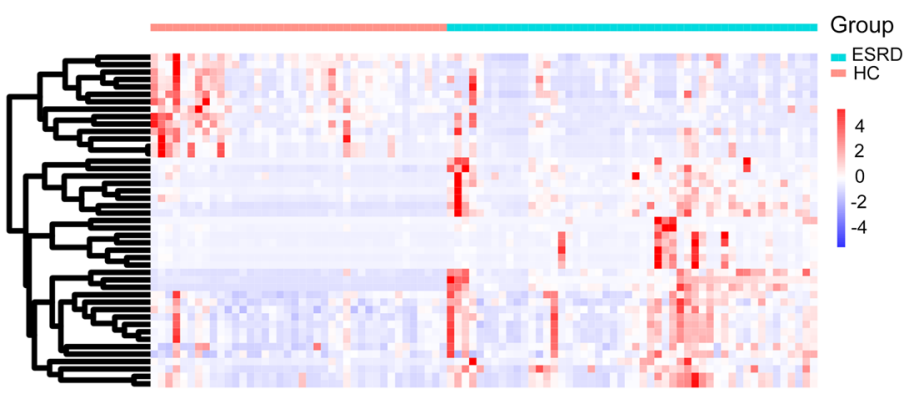

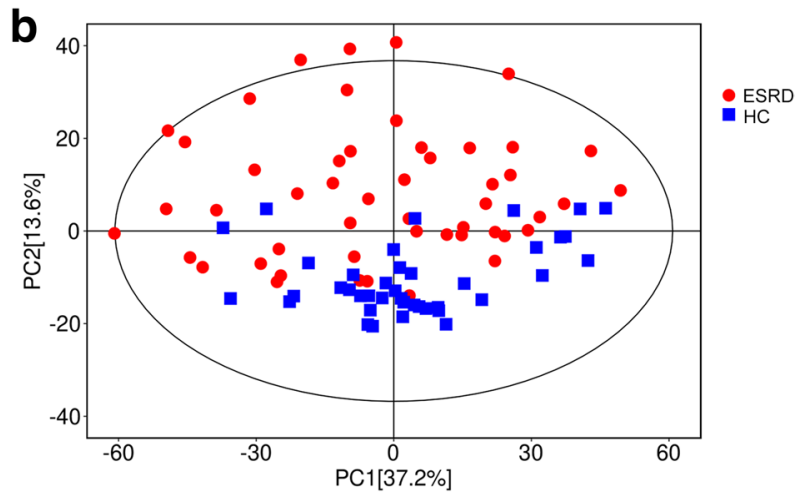

d Intercepts: $R^{2} Y($ cum $)=(0,-0.09), Q^{2}($ cum $)=(0,-1)$

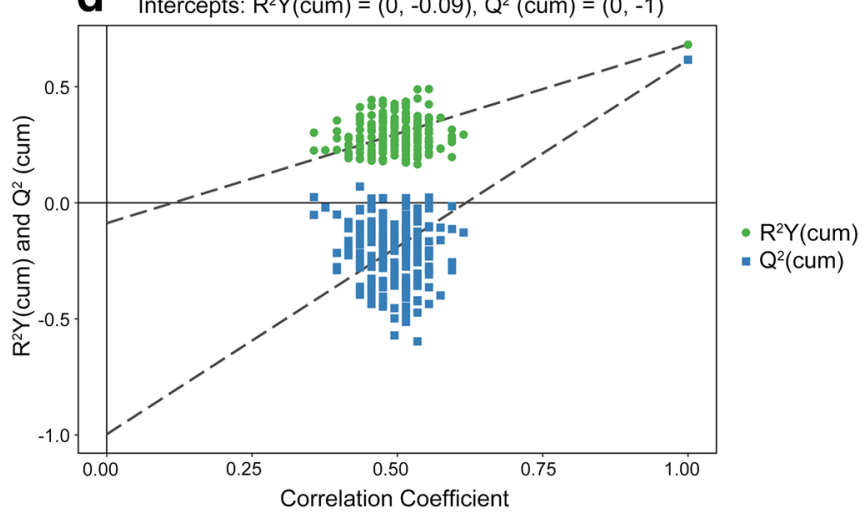

Fig. 2 Metabolic profile of GCF based on LC-MS. a Heatmap of metabolites between ESRD group and HC group. b PCA score plot model of GCF samples. c OPLS-DA score plot of GCF samples. d OPLS-DA permutation plot of GCF samples. LC-MS, liquid chro-

\section{Result interpretation of IL-8 in the pathogenesis of periodontal disease}

According to the 2018 American Academy of Periodontology and European Federation of Periodontology, periodontal health would be defined as a clinically non-inflammatory status, which means that absence of inflammation in periodontal tissue is a prerequisite for defining periodontal health [32]. And the success of treatment for periodontitis is to minimize inflammation and improve periodontal parameters [32]. We utilized inflammatory markers, especially IL-8, to reflect the periodontal inflammation status between the groups with and without ESRD. IL-8 can help neutrophils migrate from gingival tissue to the gingival crevice, thus facilitates the inflammatory cell infiltration and release of granule enzymes to efficiently degrade periodontal connective tissue [20,33]. Previous studies proved that IL-8 was mainly detected in deeper layers of the pocket epithelium and indicated that IL-8 was involved in the induction and development of periodontal disease [21, 34, 35]. Dag A et al. reported that IL-8 levels in GCF were found higher in hemodialysis patients compared with the healthy individuals [36]. The finding was consistent with our study. IL-8 matography/mass spectrometry; ESRD, end-stage renal disease; HC, healthy controls; OPLS-DA, orthogonal partial least-squares discriminant analysis; PCA, principal component analysis

may play an important role in the periodontal condition of ESRD patients.

\section{Metabolite as a tool for predicting periodontal inflammation status}

When periodontal tissue is in an inflammatory state, inflammatory factors such as cytokines, bacterial antigens, various cells, metabolites, and other degradation products are released in the GCF [37]. Interestingly, IL-8 showed a significant positive correlation with taurine and 3 -sulfinoalanine in our study. Taurine, a type of free amino acid with antimicrobial and antioxidant activities, has a great impact on important biological processes related to inflammation [38, 39]. It was reported that taurine could inhibit oxidation of hypochloric acid associated with myeloperoxidase-hydrogen peroxide-halide system of neutrophils [38]. 3-Sulfinoalanine, a product of cysteine dioxygenase, is involved in several processes, such as pyruvate production and taurine/hypotaurine synthesis [40]. The finding suggests that abnormally elevated inflammatory markers may induce the production of taurine and 3-sulfinoalanine to inhibit the inflammation. We also found that other significant metabolites of GCF were 
Table 5 Differential metabolites of GCF between patients with ESRD and healthy controls

\begin{tabular}{|c|c|c|c|c|c|c|}
\hline Metabolic pathway & Metabolites & Mean (ESRD) & Mean $(\mathrm{HC})$ & Fold change & Log_fold change & AUC value \\
\hline \multirow[t]{2}{*}{ Nicotinate and nicotinamide metabolism } & Niacinamide & 0.0361 & 0.1008 & 0.359 & -1.479 & 0.703 \\
\hline & $\begin{array}{l}\text { N1-Methyl-2-pyri- } \\
\text { done-5-carboxa- } \\
\text { mide }\end{array}$ & 0.3889 & 0.0505 & 7.701 & 2.945 & 0.952 \\
\hline \multirow[t]{2}{*}{ Phenylalanine metabolism } & $m$-Coumaric acid & 1.5604 & 2.4824 & 0.629 & -0.670 & 0.748 \\
\hline & Hydrocinnamic acid & 10.0707 & 32.8998 & 0.306 & -1.708 & 0.823 \\
\hline \multirow[t]{2}{*}{ Taurine and hypotaurine metabolism } & Taurine & 5.7182 & 9.2706 & 0.617 & -0.697 & 0.778 \\
\hline & 3-Sulfinoalanine & 1.1994 & 2.0963 & 0.572 & -0.806 & 0.747 \\
\hline \multirow[t]{3}{*}{ Purine metabolism } & Inosine & 0.1574 & 0.3363 & 0.468 & -1.095 & 0.843 \\
\hline & Xanthine & 0.9294 & 1.2673 & 0.733 & -0.447 & 0.687 \\
\hline & Hypoxanthine & 26.5315 & 42.3163 & 0.627 & -0.674 & 0.768 \\
\hline \multirow[t]{2}{*}{ Galactose metabolism } & alpha-D-Glucose & 0.0889 & 0.0099 & 8.961 & 3.164 & 0.714 \\
\hline & Raffinose & 0.0408 & 0.0013 & 32.389 & 5.017 & 0.740 \\
\hline Starch and sucrose metabolism & D-Maltose & 0.5640 & 0.0761 & 7.413 & 2.890 & 0.744 \\
\hline \multirow[t]{2}{*}{ Pyrimidine metabolism } & Uridine & 2.9903 & 5.2445 & 0.570 & -0.810 & 0.793 \\
\hline & dUMP & 0.0358 & 0.0041 & 8.692 & 3.120 & 0.709 \\
\hline
\end{tabular}

$G C F$, gingival crevicular fluid; $E S R D$, end-stage renal disease; $H C$, healthy controls; $A U C$, area under curve

more abundant in hemodialysis patients than in the healthy controls. However, the mechanism is not clear due to a shortage of the relevant researches.

\section{Discrepancies between serum and GCF with regard to metabolites and inflammatory markers}

Furthermore, though the compositions of GCF mainly originated from serum [37], there were many discrepancies between serum and GCF with regard to metabolites and inflammatory markers. IL-6 levels were significantly different in the serum between two groups rather than IL-8 levels. IL-6-associated and CRP-associated metabolites including pseudouridine, L-phenylalanine, and p-hydroxyphenylacetic acid were reported to be associated with renal

Table 6 Correlation between various inflammatory markers and key metabolites in serum and GCF between the two groups

\begin{tabular}{lll}
\hline & ESRD group & HC group \\
\hline$p$-Hydroxyphenylacetic acid and IL-6 & $0.519^{* *}$ & -0.081 \\
L-Phenylalanine and IL-6 & $0.492^{* *}$ & -0.146 \\
Pseudouridine and IL-6 & $0.377^{* *}$ & -0.132 \\
$p$-Hydroxyphenylacetic acid and CRP & $0.324^{*}$ & $0.309^{*}$ \\
L-Phenylalanine and CRP & $0.309^{*}$ & $0.507^{* *}$ \\
Taurine and IL-8 & $0.570^{* *}$ & $0.416^{* *}$ \\
3-Sulfinoalanine and IL-8 & $0.469^{* *}$ & 0.016 \\
\hline
\end{tabular}

$E S R D$, end-stage renal disease; $H C$, healthy controls; $G C F$, gingival crevicular fluid; $I L-6$, interleukin-6; $I L-8$, interleukin-8; $C R P$, C-reactive protein

${ }^{*} P<0.05$

${ }^{* *} P<0.01$ disease [41-43], which were seen obviously abundant in the serum instead of GCF in the ESRD patients. The discrepancies between serum and GCF exist due to the interaction of host defense, oral microbial species, and external factors [37]. Evidence supports ESRD could make periodontal microbial community different from that of those without ESRD [44]. The interplay between microbial agent and the adjacent periodontal tissues often increases gingival inflammation and releases biologically active substances [45].

\section{PPPM concept in the current study}

Early prediction and prevention of diseases using precise monitoring methods is a key paradigm in healthcare [46]. GCF, a kind of non-invasive tissue fluid and easily processed, contains tremendous metabolites from the host as well as bacteria [47, 48]. GCF is likely to reflect the periodontal health status [48]. ESRD patients are susceptible to infection due to the impairment of immune system, leading to an increase of proinflammatory cytokines [11]. Our study indicated that there were some correlations between key metabolites and inflammation. Therefore, the inflammatory markers and metabolites in GCF may be valuable biomarkers for patient stratification, disease surveillance, predictive diagnosis, and targeted prevention. Through the omics analysis of GCF, we can determine unique metabolite information and then analyze the related molecular pathways in global terms, which can predict whether there is any disorder in periodontal tissue or system health [49]. Specifically, based on the results in this study, the individuals may be in a state of periodontal inflammation and at a risk of periodontal 
disease when the most direct biomarkers such as taurine in GCF decreased greatly compared with the previous levels or were at a low level. The prediction can trigger targeted preventions, such as increased attention to oral hygiene (e.g., the frequency of tooth brushing) and regular oral care (e.g., supragingival scaling) [50]. Meanwhile, the metabolites with anti-inflammatory and antioxidant properties found in this study can aid in understanding the pathogenesis of periodontal disease of ESRD patients and help in the development of therapeutics.

\section{Strengths and limitations}

Some limitations of this study need to be addressed. Selection bias may exist as the proportions of participants with the history of diabetes and hypertension were uneven in the two groups; The bias is difficult to avoid as diabetes and hypertension are the leading causes for ESRD, account for $61 \%$ of dialysis cases [51-53]. However, multiple factor analysis was performed to analyze the associations between the inflammatory marker and these two variables. And the result showed hypertension and diabetes were not significantly associated with the IL-8 levels of GCF. Secondly, clinical attachment loss was not included in periodontal parameters of the study as it was challenging and time-consuming to measure gingival recession and cemento-enamel junction in the chairside operation [54]. Much work has already been done to focus on the interaction between ESRD and periodontal health. However, the relationship between periodontal and kidney diseases has not been explored deeply so far. Our results provide some clues regarding their relationships based on significant differences of inflammatory markers and metabolites. To the best of our knowledge, it is the first study that reported the metabolites and its association with inflammation in the GCF of ESRD patients, the extent to discuss these findings is limited for us. The role of the hemodialysis may lead to the poor periodontal health status [6]. However, further evidence is certainly needed to draw causal conclusions in the future.

\section{What is known about liquid biopsy in COVID-19 management?}

The novel Coronavirus Disease 2019 (COVID-19) is the most dramatic healthcare crisis that has triggered a significant shock in the worldwide economy with quarantine policies [55]. It was reported that CKD seemed to be associated with enhanced risk of severe COVID-19 infection [56]. During the COVID-19 pandemic, it is a great challenge for the management of CKD patients needing dialysis treatment at the hospital as they cannot respect the quarantine, and there is no guarantee that they correctly follow the preventive measures at home [57]. Furthermore, they may ignore oral health care and other potential problems due to the length of time spent in the dialysis center, leading to the delay of diagnosis and treatment of some oral diseases like periodontitis [36]. Patients with CKD should hence be advised to take extra non-invasive and simple methods to minimize risk of the exposure to other resource infection. As a non-invasive diagnostic method, liquid biopsy can obtain lots of information about disease from liquid samples, such as GCF. Detection of inflammatory and metabolic markers is highly indicative for periodontal health status of individuals, especially for those ESRD patients with compromised immunity.

\section{Conclusions}

The periodontal health status of ESRD patients appears to be worse than the average individuals. Inflammation markers including IL-8 and CRP were significantly higher in the GCF of ESRD patients, which can be indicators of periodontal health status and may be considered the valuable predictive diagnostics especially in the ESRD patients, who are susceptible to the infection due to systemic immune disorders. Besides, metabolites of taurine in GCF as well as L-phenylalanine and $p$-hydroxyphenylacetic acid in serum are possible biomarkers correlated with inflammatory cytokine. All these biomarkers may also be highly recommended as a novel predictive/diagnostic tool for the assessment of inflammation status from the perspectives of PPPM in view of susceptible population and individual screening. And some metabolites such as taurine with antiinflammatory and antioxidant properties can be applied as the targeted medicine to the patient in focus in the agreement with PPPM philosophy. Integration of inflammatory and metabolic markers in routine medical checks as a prehealth screening and judgement of disease progression will create opportunity for risk assessment, disease stratification, individualized management, and targeted preventive measures, which are all key concepts in PPPM.

Acknowledgements We thank Wei Lu for her help in collecting serum samples.

Author contribution Xiaoxin Ma contributed to the study conception and design, data analysis, and drafted the manuscript. Yongli Wang contributed to the study conception and design, and drafted the manuscript. Hongyu Wu, Fei Li, Yingxin Xie, Danshu Xie, and Wenji Wang contributed to the sample collection. Xiping Feng and Edward. Chin. Man. Lo contributed to the study conception and design, and critically revised the manuscript. Haixia Lu contributed to the literature review, study conception and design, and data analysis, and drafted the manuscript. All authors read and approved the final manuscript. 
Funding This work was supported by the Shanghai Municipal Key Clinical Specialty (shslczdzk01601), Shanghai Clinical Research Center for Oral Diseases(19MC1910600), and Clinical Research Program of Ninth People's Hospital, Shanghai JiaoTong University School of Medicine (201918069).

Data availability The datasets used or analyzed during the current study are available from the corresponding author on reasonable request.

Code availability All software applications used are included in this article.

\section{Declarations}

Ethics approval All procedures performed in studies involving human participants were in accordance with the ethical standards of the Institutional Ethics Committee of Ninth People's Hospital, Shanghai Jiao Tong University School of Medicine (Ethical approval number: SH9H-2018-T32-3), and with the 1964 Helsinki Declaration and its later amendments or comparable ethical standards.

Consent to participate The protocol was established, according to the ethical guidelines of the Helsinki Declaration, and was approved by the Institutional Ethics Committee of Ninth People's Hospital, Shanghai Jiao Tong University School of Medicine. Written informed consent was obtained from individual or guardian participants.

Consent for publication Not applicable.

Conflict of interest The authors declare no competing interests.

Open Access This article is licensed under a Creative Commons Attribution 4.0 International License, which permits use, sharing, adaptation, distribution and reproduction in any medium or format, as long as you give appropriate credit to the original author(s) and the source, provide a link to the Creative Commons licence, and indicate if changes were made. The images or other third party material in this article are included in the article's Creative Commons licence, unless indicated otherwise in a credit line to the material. If material is not included in the article's Creative Commons licence and your intended use is not permitted by statutory regulation or exceeds the permitted use, you will need to obtain permission directly from the copyright holder. To view a copy of this licence, visit http://creativecommons.org/licenses/by/4.0/.

\section{References}

1. Romagnani P, Remuzzi G, Glassock R, Levin A, Jager KJ, Tonelli M, et al. Chronic kidney disease. Nat Rev Dis Primers. 2017;3:17088.

2. Nagai K, Matsuura M, Tsuchida K, Kanayama HO, Doi T, Minakuchi J. Prognostic factors for mortality in middle-aged and older hemodialysis patients a 5-year observational study. J Artif Organs. 2018;21(1):94-101.

3. Cholewa M, Madziarska K, Radwan-Oczko M. The association between periodontal conditions, inflammation, nutritional status and calcium-phosphate metabolism disorders in hemodialysis patients. J Appl Oral Sci. 2018;26:e20170495.

4. Chen LP, Chiang CK, Peng YS, Hsu SP, Lin CY, Lai CF, et al. Relationship between periodontal disease and mortality in patients treated with maintenance hemodialysis. Am J Kidney Dis. 2011;57(2):276-82.

5. Brito F, Almeida S, Figueredo CM, Bregman R, Suassuna JH, Fischer RG. Extent and severity of chronic periodontitis in chronic kidney disease patients. J Periodontal Res. 2012;47(4):426-30.

6. Borawski J, Wilczyńska-Borawska M, Stokowska W, Myśliwiec $M$. The periodontal status of pre-dialysis chronic kidney disease and maintenance dialysis patients. Nephrol Dial Transplant. 2007;22(2):457-64.

7. Kapellas K, Singh A, Bertotti M, Nascimento GG, Jamieson LM. Periodontal and chronic kidney disease association: a systematic review and meta-analysis. Nephrology (Carlton). 2019;24(2):202-12.

8. Thoppay J, Desai B. Oral burning: local and systemic connection for a patient-centric approach. EPMA J. 2019;10(1):1-11.

9. Alvarenga L, Cardozo L, Lindholm B, Stenvinkel P, Mafra D. Intestinal alkaline phosphatase modulation by food components: predictive, preventive, and personalized strategies for novel treatment options in chronic kidney disease. EPMA J. 2020;11(4):565-79.

10. Maturo MG, Soligo M, Gibson G, Manni L, Nardini C. The greater inflammatory pathway-high clinical potential by innovative predictive, preventive, and personalized medical approach. EPMA J. 2020;11(1):1-16.

11. Syed-Ahmed M, Narayanan M. Immune dysfunction and risk of infection in chronic kidney disease. Adv Chronic Kidney Dis. 2019;26(1):8-15.

12. Heine GH, Ortiz A, Massy ZA, Lindholm B, Wiecek A, MartínezCastelao A, et al. Monocyte subpopulations and cardiovascular risk in chronic kidney disease. Nat Rev Nephrol. 2012;8(6):362-9.

13. Anders HJ, Andersen K, Stecher B. The intestinal microbiota, a leaky gut, and abnormal immunity in kidney disease. Kidney Int. 2013;83(6):1010-6.

14. Akar H, Akar GC, Carrero JJ, Stenvinkel P, Lindholm B. Systemic consequences of poor oral health in chronic kidney disease patients. Clin J Am Soc Nephrol. 2011;6(1):218-26.

15. Chambrone L, Foz AM, Guglielmetti MR, Pannuti CM, Artese HP, Feres M, et al. Periodontitis and chronic kidney disease: a systematic review of the association of diseases and the effect of periodontal treatment on estimated glomerular filtration rate. J Clin Periodontol. 2013;40(5):443-56.

16. Goutoudi P, Diza E, Arvanitidou M. Effect of periodontal therapy on crevicular fluid interleukin-6 and interleukin-8 levels in chronic periodontitis. Int J Dent. 2012;2012:362905.

17. Chung RM, Grbíc JT, Lamster IB. Interleukin- 8 and betaglucuronidase in gingival crevicular fluid. J Clin Periodontol. 1997;24(3):146-52.

18. Konopka L, Pietrzak A, Brzezińska-Błaszczyk E. Effect of scaling and root planing on interleukin-1 $\beta$, interleukin- 8 and MMP-8 levels in gingival crevicular fluid from chronic periodontitis patients. J Periodontal Res. 2012;47(6):681-8.

19. Groeger SE, Meyle J. Epithelial barrier and oral bacterial infection. Periodontol 2000. 2015;69(1):46-67.

20. Bickel M. The role of interleukin- 8 in inflammation and mechanisms of regulation. J Periodontol. 1993;64(5 Suppl):456-60.

21. Andia DC, de Oliveira NF, Letra AM, Nociti FH Jr, Line SR, de Souza AP. Interleukin-8 gene promoter polymorphism (rs4073) may contribute to chronic periodontitis. J Periodontol. 2011;82(6):893-9.

22. César-Neto JB, Duarte PM, de Oliveira MC, Tambeli CH, Sallum EA, Nociti FH Jr. Smoking modulates interleukin-6:interleukin-10 and RANKL:osteoprotegerin ratios in the periodontal tissues. J Periodontal Res. 2007;42(2):184-91. 
23. Elabdeen HR, Mustafa M, Szklenar M, Rühl R, Ali R, Bolstad AI. Ratio of pro-resolving and pro-inflammatory lipid mediator precursors as potential markers for aggressive periodontitis. PLoS ONE. 2013;8(8):e70838.

24. Chen HW, Zhou W, Liao Y, Hu SC, Chen TL, Song ZC. Analysis of metabolic profiles of generalized aggressive periodontitis. J Periodontal Res. 2018;53(5):894-901.

25. Levy Y. Oxidative stress, antioxidants and periodontal disease. Arch Oral Biol. 2015;60(9):1461-2.

26. Barnes VM, Kennedy AD, Panagakos F, Devizio W, Trivedi HM, Jönsson T, et al. Global metabolomic analysis of human saliva and plasma from healthy and diabetic subjects, with and without periodontal disease. PLoS ONE. 2014;9(8):e105181.

27. Takahashi N, Washio J, Mayanagi G. Metabolomics of supragingival plaque and oral bacteria. J Dent Res. 2010;89(12):1383-8.

28. Moreau R, Clària J, Aguilar F, Fenaille F, Lozano JJ, Junot C, et al. Blood metabolomics uncovers inflammation-associated mitochondrial dysfunction as a potential mechanism underlying ACLF. J Hepatol. 2020;72(4):688-701.

29. Sekiguchi RT, Pannuti CM, Silva HT Jr, Medina-Pestana JO, Romito GA. Decrease in oral health may be associated with length of time since beginning dialysis. Spec Care Dentist. 2012;32(1):6-10.

30. Kanjanabuch P, Sinpitaksakul P, Chinachatchawarat S, Pacharapong S, Kanjanabuch T. Oral and radiographic findings in patients undergoing continuous ambulatory peritoneal dialysis. J Med Assoc Thai. 2011;94(Suppl 4):S106-12.

31. Teratani G, Awano S, Soh I, Yoshida A, Kinoshita N, Hamasaki T, et al. Oral health in patients on haemodialysis for diabetic nephropathy and chronic glomerulonephritis. Clin Oral Investig. 2013;17(2):483-9.

32. Lang NP, Bartold PM. Periodontal health. J Clin Periodontol. 2018;45(Suppl 20):S9-s16.

33. Tonetti MS, Imboden MA, Lang NP. Neutrophil migration into the gingival sulcus is associated with transepithelial gradients of interleukin-8 and ICAM-1. J Periodontol. 1998;69(10):1139-47.

34. Finoti LS, Nepomuceno R, Pigossi SC, Corbi SC, Secolin R, Scarel-Caminaga RM. Association between interleukin-8 levels and chronic periodontal disease: a PRISMA-compliant systematic review and meta-analysis. Medicine (Baltimore). 2017;96(22):e6932

35. Tonetti MS, Imboden MA, Gerber L, Lang NP, Laissue J, Mueller C. Localized expression of mRNA for phagocyte-specific chemotactic cytokines in human periodontal infections. Infect Immun. 1994;62(9):4005-14.

36. Dağ A, Firat ET, Kadiroğlu AK, Kale E, Yilmaz ME. Significance of elevated gingival crevicular fluid tumor necrosis factor-alpha and interleukin-8 levels in chronic hemodialysis patients with periodontal disease. J Periodontal Res. 2010;45(4):445-50.

37. Barros SP, Williams R, Offenbacher S, Morelli T. Gingival crevicular fluid as a source of biomarkers for periodontitis. Periodontol 2000. 2016;70(1):53-64.

38. Marcinkiewicz J, Kontny E. Taurine and inflammatory diseases. Amino Acids. 2014;46(1):7-20.

39. Maleki V, Mahdavi R, Hajizadeh-Sharafabad F, Alizadeh M. The effects of taurine supplementation on oxidative stress indices and inflammation biomarkers in patients with type 2 diabetes: a randomized, double-blind, placebo-controlled trial. Diabetol Metab Syndr. 2020;12:9.

40. Zhu SS, Long R, Song T, Zhang L, Dai YL, Liu SW, et al. UPLC-Q-TOF/MS based metabolomics approach to study the hepatotoxicity of cantharidin on mice. Chem Res Toxicol. 2019;32(11):2204-13.

41. Sriboonvorakul N, Ghose A, Hassan MMU, Hossain MA, Faiz MA, Pukrittayakamee S, et al. Acidosis and acute kidney injury in severe malaria. Malar J. 2018;17(1):128.
42. Hocher B, Adamski J. Metabolomics for clinical use and research in chronic kidney disease. Nat Rev Nephrol. 2017;13(5):269-84.

43. Kopple JD. Phenylalanine and tyrosine metabolism in chronic kidney failure. J Nutr 2007;137(6 Suppl 1):1586S-1590S; discussion 1597S-1598S.

44. Araújo MV, Hong BY, Fava PL, Khan S, Burleson JA, Fares G, et al. End stage renal disease as a modifier of the periodontal microbiome. BMC Nephrol. 2015;16:80.

45. Altamimi AG, AlBakr SA, Alanazi TA, Alshahrani FA, Chalisserry EP, Anil S. Prevalence of periodontitis in patients undergoing hemodialysis: a case control study. Mater Sociomed. 2018;30(1):58-61.

46. Seredin P, Goloshchapov D, Ippolitov Y, Vongsvivut P. Pathology-specific molecular profiles of saliva in patients with multiple dental caries-potential application for predictive, preventive and personalised medical services. EPMA J. 2018;9(2):195-203.

47. Yu JC, Khodadadi H, Baban B. Innate immunity and oral microbiome: a personalized, predictive, and preventive approach to the management of oral diseases. EPMA J. 2019;10(1):43-50.

48. Ozeki M, Nozaki T, Aoki J, Bamba T, Jensen KR, Murakami $\mathrm{S}$, et al. Metabolomic analysis of gingival crevicular fluid using gas chromatography/mass spectrometry. Mass Spectrom (Tokyo). 2016;5(1):A0047.

49. Pei J, Li F, Xie Y, Liu J, Yu T, Feng X. Microbial and metabolomic analysis of gingival crevicular fluid in general chronic periodontitis patients: lessons for a predictive, preventive, and personalized medical approach. EPMA J. 2020;11(2):197-215.

50. Kunin A, Polivka J Jr, Moiseeva N, Golubnitschaja O. "Dry mouth" and "Flammer" syndromes-neglected risks in adolescents and new concepts by predictive, preventive and personalised approach. EPMA J. 2018;9(3):307-17.

51. Ng YP, Ahmed R, Ooi GS, Lau CY, Balasubramanian GP, Yap $\mathrm{CH}$. The rate of progression of type 2 diabetes mellitus to end stage renal disease - a single centred retrospective study from Malaysia. Diabetes Metab Syndr. 2018;12(6):1025-30.

52. Prischl FC, Auinger M, Säemann M, Mayer G, Rosenkranz AR, Wallner M, et al. Diabetes-related end-stage renal disease in Austria 1965-2013. Nephrol Dial Transplant. 2015;30(11):1920-7.

53. Kim YJ, Moura LM, Caldas CP, Perozini C, Ruivo GF, Pallos D. Evaluation of periodontal condition and risk in patients with chronic kidney disease on hemodialysis. Einstein (Sao Paulo). 2017;15(2):173-7.

54. Chang JF, Yeh JC, Chiu YL, Liou JC, Hsiung JR, Tung TH. Periodontal pocket depth, hyperglycemia, and progression of chronic kidney disease: a population-based longitudinal study. Am J Med. 2017;130(1):61-69.e1.

55. Radanliev P, De Roure D, Walton R, Van Kleek M, Montalvo RM, Santos O, et al. COVID-19 what have we learned? The rise of social machines and connected devices in pandemic management following the concepts of predictive, preventive and personalized medicine. EPMA J. 2020;11(3):311-32.

56. Henry BM, Lippi G. Chronic kidney disease is associated with severe coronavirus disease 2019 (COVID-19) infection. Int Urol Nephrol. 2020;52(6):1193-4.

57. Rombolà G, Brunini F. COVID-19 and dialysis: why we should be worried. J Nephrol. 2020;33(3):401-3.

Publisher's Note Springer Nature remains neutral with regard to jurisdictional claims in published maps and institutional affiliations. 